\title{
Ask Suicide-Screening Questions to Everyone in Medical Settings: The asQ'em Quality Improvement Project
}

\author{
Lisa M. Horowitz, Ph.D., M.P.H., Deborah Snyder, M.S.W., Erica Ludi, B.S., Donald L. \\ Rosenstein, M.D., Julie Kohn-Godbout, M.S.N., R.N., PMHCNS-BC, Laura Lee, R.N., B.S.N., \\ Tannia Cartledge, R.N., M.S., Adrienne Farra, M.S.W., Ph.D., and Maryland Pao, M.D. \\ Office of the Clinical Director, National Institute of Mental Health, National Institutes of Health, \\ Bethesda, MD (Drs. Horowitz \& Pao, Ms. Snyder, Ms. Ludi); Department of Nursing, Clinical \\ Center, National Institutes of Health, Bethesda, MD (Ms. Kohn-Godbout, Ms. Lee, Ms. Cartledge); \\ Department of Social Work, Clinical Center, National Institutes of Health, Bethesda, MD (Dr. \\ Farrar); Comprehensive Cancer Support Program, University of North Carolina at Chapel Hill, \\ Chapel Hill, NC (Dr. Rosenstein).
}

\begin{abstract}
Background-Suicide in hospital settings is a frequently reported sentinel event to the Joint Commission (JC). Since 1995, over 1,000 inpatient deaths by suicide have been reported to the JC; $25 \%$ occurred in non-behavioral health settings. Lack of proper "assessment" was the leading root cause for $80 \%$ of hospital suicides. This paper describes the "Ask Suicide-Screening Questions to Everyone in Medical Settings (asQ'em)" Quality Improvement Project. We aimed to pilot a suicide screening tool and determine feasibility of screening in terms of prevalence, impact on unit workflow, impact on mental health resources, and patient/nurse acceptance.
\end{abstract}

\begin{abstract}
Methods-We piloted the asQ'em 2-item screening instrument that assesses suicidal thoughts and behaviors, designed specifically for nurses to administer to medical patients. Educational inservices were conducted. A convenience sample of adult patients 18 years or older, from three selected inpatient units in the National Institutes of Health Clinical Center, participated.
\end{abstract}

\begin{abstract}
Results-331 patients were screened. 13 (4\%) patients screened "positive" for suicide risk and received further evaluation. No patient had acute suicidal thoughts or required an observational monitor. Screening took approximately 2 minutes; $87 \%$ of patients reported feeling comfortable with screening. $81 \%$ of patients, $75 \%$ of nurses, and $100 \%$ of social workers agreed that all patients in hospitals should be screened for suicide risk.
\end{abstract}

Discussion-Nurses can feasibly screen hospitalized medical/surgical patients for suicide risk with a 2-item screening instrument. Patients, nurses, and social workers rated their experience of screening as positive, and supported the idea of universal suicide screening in the hospital.

\footnotetext{
(C) 2013 Academy of Psychosomatic Medicine. Published by Elsevier Inc. All rights reserved.

Corresponding Author: Lisa M. Horowitz, PhD, MPH, National Institute of Mental Health, Clinical Research Center, Building 10, Room 6-5362, Bethesda, MD 20892, Phone: 301-435-6052, Fax 301-402-2588, horowitzl@ mail.nih.gov.

Publisher's Disclaimer: This is a PDF file of an unedited manuscript that has been accepted for publication. As a service to our customers we are providing this early version of the manuscript. The manuscript will undergo copyediting, typesetting, and review of the resulting proof before it is published in its final citable form. Please note that during the production process errors may be discovered which could affect the content, and all legal disclaimers that apply to the journal pertain.

Disclosures

The opinions expressed in the article are the views of the authors and do not reflect the views of the Department of Health and Human Services or the United States government. The authors have no relevant financial interests in this manuscript to disclose.
} 


\section{Keywords}

suicide screening; medical patients / surgical patients; adults; risk of suicide; suicidal ideation; suicidal behavior; risk for suicide

\section{INTRODUCTION}

Suicide in a hospital setting is one of the most frequent sentinel events reported to the Joint Commission (JC). ${ }^{1}$ Since 1995, over 1,000 patient deaths by suicide have been reported to the JC from hospitals across the country. ${ }^{2}$ Notably, $25 \%$ of these suicides occurred in nonbehavioral health settings such as oncology units and the emergency department. Lack of proper "assessment" of suicide risk was the leading root cause for $80 \%$ of the reported suicides. $^{3}$

There is a growing body of evidence suggesting that medical patients are at increased risk for suicide, with greater prevalence in cancer and cardiac patients. ${ }^{4-7}$ Early detection of patients at elevated risk for suicide is a critical suicide prevention strategy, ${ }^{8-10}$ yet high-risk patients are often not recognized by healthcare providers. ${ }^{11-13}$

In November 2010, the JC issued a "Sentinel Event Alert," highlighting the need for suicide risk screening and treatment for all patients in non-behavioral health settings. ${ }^{14}$ Non-mental health clinicians on the frontlines will require training and standardized instruments to screen patients for suicide risk and safely manage those who screen positive.

While many hospitals are working hard to comply with the JC alert and implement suicide screening questions, there are limited data available to guide suicide risk screening initiatives in a general medical setting ${ }^{15-17}$. As part of a two-phase Quality Improvement Project (QIP), spearheaded by the Psychiatry Consultation-Liaison Service (PCLS) at the National Institutes of Health (NIH), we set out to develop a process for nurses to screen patients for suicide risk on inpatient medical/surgical units in a hospital. Nurses were selected because they have the most patient contact and routinely screen patients for other risk factors during admission.

This paper describes the "Ask Suicide-Screening Questions to Everyone in Medical Settings (asQ'em)" QIP. We aimed to: 1) pilot a suicide screening tool designed for medical/surgical adult inpatients; 2) determine feasibility of suicide screening in three domains: prevalence, impact on unit workflow, and patient/nurse acceptance; and 3) describe suicide screening impact on mental health resources.

\section{METHODS}

The QIP was created with a traditional "plan-do-study-act" approach. ${ }^{20}$ Inclusion of nursing administration and staff, physician leaders, social work department, and senior hospital administrators throughout the process was critical to acceptance and delivery of the QIP. The NIH Clinical Center (CC) nursing and social work administrative leadership and the Medical Executive Committee all participated in decision-making for the QIP guidelines. The "asQ'em team" was composed of a psychiatrist, a nurse champion, a PCLS psychologist and clinical social worker (SW), and a research assistant.

In order to ensure nursing uptake and patient acceptance, and to stay true to the dynamic process of "plan do study act," the QIP was completed in two phases: the goal of the first week (Phase I) was to pilot the screening instrument and assess nurse/patient reaction to 
suicide screening; the goal of the subsequent 8 weeks (Phase II) was to investigate the impact of positive screens on mental health resources.

\section{Setting and Population}

The NIH CC is a 200-bed research hospital with over 6,000 inpatient admissions and 100,000 outpatient visits a year. The convenience sample was comprised of patients, 18 years or older, enrolled in a medical/surgical clinical research trial at the NIH CC who were admitted during designated data collection weeks (Phase I: January 8-12; Phase II: April 23June 18, 2012) on three selected inpatient units. Two units, a cancer unit and an infectious disease unit, were chosen to participate in the QIP because they were frequent users of the PCLS; the third unit was chosen because it was a general medical/surgical unit. Patients were excluded if they were unable to fluently communicate in English, cognitively impaired, or their medical status worsened acutely. This QIP was determined to be "exempt" from IRB review by the NIH Office of Human Subjects Research.

\section{Measures}

The asQ'em Screening Instrument-The Ask Suicide-Screening Questions to Everyone in Medical Settings (asQ'em) screening instrument consists of a brief script and 2 questions (see Figure). The script was developed to provide standardized language to minimize nurse and patient discomfort in transitioning from a medically-oriented nursing review of systems to questions of a more personal and sensitive nature. The questions were adapted from published literature ${ }^{21-22}$, experiences from the Veterans Affairs clinical care system and other hospitals, and expert opinion from health service researchers and clinicians trained to assess for risk of suicide. The questions assess two of the most critical risk factors for completed suicide, present thoughts and past behavior: 1) "In the past month, have you had thoughts about suicide?" 2) "Have you ever made a suicide attempt?" If the patient answered yes to either question, a follow-up question was asked, "Are you having thoughts of suicide right now?" We chose to screen for suicidal thoughts and behaviors because they are not only predictive of completed suicide, ${ }^{18-19}$ but are markers of significant emotional distress warranting further mental health attention.

Patient, Nurse, and Social Work Feedback Surveys-Patients were asked to rate their experience of being screened in several domains, including their comfort level with being asked about suicidal thoughts and behaviors. Patients were also given the opportunity to describe their experience of being screened and to give feedback about the overall process. The nurses were also asked to complete a feedback survey of their experience after screening each patient. They were asked to rate items such as their own comfort in assessing the patient for suicide, their perception of the patient's comfort in answering, and the ease of administering the asQ'em instrument. SWs filled out similar surveys with questions about their comfort level in evaluating patients who screened positive for suicide risk, how accurate the asQ'em questions were in detecting risk, and their opinions about suicide screening in medical settings (see Appendix).

\section{Suicide Risk Training}

Educational in-services were conducted for nurses, physicians, SWs and other licensed individual practitioners prior to data collection weeks. In addition, training was also offered to unit nurses on data collection days. Fifty-three unit nurses participated in the formal educational sessions. In order to bridge the communication gap to which hospitals are vulnerable, a PCLS psychiatrist from the asQ'em team attended service rounds for each physician group associated with selected units and presented educational information for approximately 20 minutes. The asQ'em curriculum included an overview of the 
epidemiology of suicide, recent JC data, clinical warning signs/risk factors, project aims, and procedural details. The education component was essential as most clinicians had limited practical experience in the assessment and management of suicidal patients.

\section{Procedures}

The asQ'em instrument was developed and piloted as standard of care for the nine week QIP period. During data collection weeks, the asQ'em team gathered daily census numbers on each of the three units. Trained unit clerks identified newly admitted/readmitted patients and created screening packets for the nurses' convenience. The admitting nurse administered asQ'em screening questions during the initial nursing assessment. Family members and friends were asked to leave the room during the brief suicide screening time in order to ensure patient privacy. All patients who were screened during this first week, regardless of suicide risk, were seen by a member of the asQ'em team who administered the patient feedback survey within 24 hours of the screening. Nurses were asked to complete a confidential nurse feedback survey shortly after screening each patient. SWs who evaluated positive screens were also asked to fill out feedback surveys. All patient, nurse and SW feedback surveys were coded, de-identified, and entered into a database. Three post-QIP feedback sessions were held with nurses from all three units in order to present the results and obtain nursing reactions and comments on the initiative.

Patients were considered "positive" risk for suicide if they answered "yes" to either question 1 or 2. If a patient screened positive, the nurse then administered the follow-up question related to current acute suicidal thoughts ("Are you having thoughts of suicide right now?"). During the first week of the QIP, all positive screens were evaluated within the hour by an "on-call" member of the NIH PCLS, who conducted full psychiatric interviews. Results were documented in the electronic medical record. Relevant clinical findings were relayed verbally to the patient's nurse and medical/surgical team.

After the first week of data collection, when the nurses and the PCLS felt more comfortable with managing patient safety in the QIP, the algorithm for responding to positive screens was changed (Phase II). For Phase II, unit SWs replaced PCLS as first responders to any positive screen (within 24 hours). However, PCLS was still "on call” for immediate response to any acute suicidal ideation. Each participant who screened positive for suicide risk received a full psychiatric evaluation to assess safety and need for short- or long-term intervention. Medical records were accessed for clinical and QI purposes in order to note whether or not suicide risk had been previously detected by the medical team. In addition, in order to minimize burden on nurses and patients, the asQ'em team only requested feedback surveys from patients who screened positive and the nurses and SWs who screened/ evaluated them.

\section{Data Analysis}

Data were reviewed and analyzed using Microsoft Excel and SPSS, version 19 for Windows. Descriptive statistics and univariate analyses were calculated.

\section{RESULTS}

337 participants were approached for screening during the QIP. Six patients were ineligible because they did not speak English fluently, had severe cognitive impairment or were receiving acute end of life treatment. 331 patients were ultimately screened for suicide risk during the initial nursing assessment upon admission to one of three units: cancer unit (63\%; $\mathrm{n}=207)$; general medicine/surgical unit $(29 \% ; \mathrm{n}=97)$ and infectious disease unit $(8 \% ; \mathrm{n}=$ 27) 
During the first week of data collection, 56 patients participated. Participants were $64 \%(\mathrm{n}=$ 36) female; $63 \%(\mathrm{n}=35)$ white and $25 \%(\mathrm{n}=14)$ black; average age was 48.1 years $( \pm 19.2 \mathrm{y}) .44 \%(\mathrm{n}=25)$ had a general medical/surgical diagnosis, $40 \%(\mathrm{n}=22)$ were cancer patients, and $16 \%(\mathrm{n}=9)$ were infectious disease patients. 53 of 56 patients $(95 \%)$ completed a feedback survey.

55 of 56 nurse feedback surveys (98\%) were returned. In order to receive the most candid feedback from the nurses, surveys were anonymous; an estimated 51 nurses completed the 55 surveys. The results are presented below in categories addressing each specific QIP aim.

\section{Prevalence: Is the frequency of suicidal ideation and behavior common enough among medical/surgical patients to warrant screening on an inpatient unit?}

Of the 331 patients screened, a total of 13 (4\%) patients screened "positive" for suicide risk . 5 of $331(1.5 \%)$ patients screened "positive" for thoughts of suicide in the past month; 8 of 331 (2.4\%) for history of a previous suicide attempt. The length of time between screening and attempt ranged from 3 months to 25 years. No patient answered affirmatively to the acute screening question, "Are you having suicidal thoughts right now?" There were no adverse events. No patients required an observational monitor (i.e., "sitter"). Of the 13 patients who screened positive, $9(69 \%)$ were on the cancer unit, $3(23 \%)$ were on the general medical/surgical unit, and $1(8 \%)$ was on the infectious disease unit. Unit positive rates for the cancer unit, general medical/surgical unit, and infectious disease unit were $4.3 \%, 3.1 \%$, and $3.7 \%$, respectively.

\section{Practicality: Can medical/surgical patients be screened for suicide risk without significantly disrupting the workflow of an inpatient unit?}

Nurses administered the 2-item asQ'em screening instrument during the initial nursing assessment. During the first data collection week, the nurses were asked to record a start and end time that included reading the script, asking the screening questions and recording the patient's responses. The average time of assessment was 2 minutes; nursing time data were missing for $17 / 56(30 \%)$ of the screenings. For patients who screened positive, the time of screening averaged 5 minutes.

\section{Patient and Nurse Experience: Do patients and nurses support suicide screening on the inpatient unit?}

Patient Feedback-Patient feedback from the surveys was generally positive (see Table 2). $87 \%$ (46/53) of patients reported that they were comfortable answering questions about suicide in general; 8\% (4/53) reported feeling neutral, and 5\% (3/53) felt uncomfortable. $79 \%(42 / 53)$ rated their experience of being screened for suicide as positive, and only 1 patient $(2 \%)$ rated it as negative. $85 \%$ (45/53) of patients reported that they were glad the hospital was asking patients about suicide. Moreover, 64\% (34/53) stated that they had never been asked about suicide in a medical setting before this screening. 77\% (41/53) reported that the nurse should be the first to ask questions about suicide. The majority of the patients reported that the questions were not burdensome (94\%), did not make them angry (98\%), or anxious (85\%). Only 1 participant reported distress about being asked questions about suicide, and that person was positive for suicide risk and answered the questions despite his discomfort.

Nurse Feedback-In response to the confidential nursing feedback surveys, 60\% (33/55) of nurses reported that they were comfortable using the asQ'em screening tool. 84\% (46/55) agreed that the asQ'em tool was easy to administer. 78\% (43/55) stated that they believed the patient was comfortable answering the suicide questions. 91\% (39/43) of the nurses believed the asQ'em screen accurately identified presence or absence of risk in the patient 
they screened. $75 \%(39 / 52)$ of nurses agreed that medical/surgical patients should be asked about suicide in a hospital.

Patients and nurses were asked to rank their own comfort as well as the perceived comfort of their respective nurse/patient. Interestingly, patients, on average, rated nurses 0.5 points more 'comfortable' asking the screening questions than the nurses rated themselves $(t(49)=$ $2.99, p<.01)$. Conversely, nurses rated patients 0.35 points less 'comfortable' answering the screening questions than the patients rated themselves $(t(51)=1.97, p=.05)$.

Social Worker Feedback-Nine Social Work Feedback Surveys were collected from each encounter with patients who screened positive during Phase II. The median time of a full psychosocial evaluation was 70 minutes. 100\% of SWs reported feeling comfortable assessing the patient for suicide risk. 75\% (6/8) of SWs stated that they agreed the patient was comfortable answering the suicide questions. Further, $100 \%$ of SWs reported they believed the asQ'em screen accurately identified the presence of suicide risk in the patients they evaluated (positive screens only). All SWs reported that universal screening in a medical setting was important.

\section{DISCUSSION}

Nurses can feasibly screen hospitalized medical/surgical patients for suicide risk with a 2item screening instrument. Screening was found to be feasible in three domains: prevalence, impact on unit workflow, and patient/nurse acceptance. $4 \%$ of the medical/surgical inpatients screened positive for suicide risk; no patient was found to be at imminent risk. Nurses spent an average of 2 minutes asking the screening questions, which was considered non-disruptive to nursing workflow. Patients, nurses, and SWs supported the idea of universal screening in the hospital.

Nursing uptake of the screening process was highly organized and well executed. Several educational in-services prepared nurses in advance for the QIP. In post-QIP feedback sessions, nurses reported feeling some discomfort with asking the screening questions. They described feeling that "it's too personal" and they felt awkward asking. A minority of nurses expressed skepticism that patients engaged in life-saving treatments would be suicidal. The nurses reported that their discomfort with asking about suicide decreased over time. Of note, in feedback surveys, patients rated the nurses more comfortable "asking" the questions than the nurses rated themselves. This indicates that, while the nurses reported feeling uncomfortable, they were willing to utilize the tool and presented themselves at ease to patients. Conversely, the nurses rated the patients significantly more anxious than the patients rated themselves. During the feedback sessions, nurses commented that perhaps they were projecting their own anxiety onto the patients.

One of the most encouraging findings was that $100 \%$ of the eligible patients agreed to participate in the QIP. The majority of patients reported that they were "glad" the hospital was asking about suicidal thoughts/behaviors, and that screening afforded an opportunity to get mental health help if needed. Three patients reported feeling uncomfortable with the screening ( 2 were positive screens). Of note, the negative feedback came mainly from one patient who screened positive. His objections stemmed from concerns about stigma and worried he would be treated differently if nurses knew about his past suicidal behavior.

Overall, screening mostly impacted mental health resources. Eight of the 13 positive screens had significant symptoms that required a full psychiatric evaluation from the PCLS; however, there were no acute cases that warranted an observational monitor or psychiatric hospitalization. Clinical SWs played a critical role in communicating with the nurses and 
care team. One important observation noted by SWs was that once a patient screened positive for suicide risk, it was difficult to integrate this important mental health information into the primary team's care plan. They commented on the absence of a "quarterback" or hospital infrastructure to continually monitor the "at risk" patient in future visits. Overall, the asQ'em initiative added more responsibility to the SWs' already full caseload; however, they reported the screening to be meaningful, and "worth the extra time." More data on the mental health resources required to effectively manage positive screens are needed.

Only one of the 13 patients who screened positive on the asQ'em tool had a documented history of suicide assessment in his medical chart. Detailed descriptions of the patients who screened positive, gathered during the psychiatric evaluations, further illuminate the value of screening. Five of the 13 patients who screened positive reported suicidal thoughts within the past month. For example, a patient reported that prior to her hospitalization, she had awakened with worry in the middle of the night and shared her thoughts with only her spouse. When the nurse screened her for suicide risk, the patient eloquently described the relief she felt in discussing her fears, which she had kept to herself. Identifying this patient as "at-risk" allowed us to refer her to a local mental health clinician who could support her through her cancer treatment and monitor future suicidal thinking.

The other eight patients screened positive for suicidal behavior at varying lengths of time in the past. Four patients attempted suicide in the past four years and had clinically relevant histories of suicidal behavior that, while not constituting an emergency, would be important to explore further for safety reasons. For example, the most serious case involved a traumatic brain injury patient who was hospitalized for removal of a neuroendocrine tumor. He had attempted suicide 3 months prior to asQ'em screening, yet did not report this to his medical care team. Unbeknownst to the team, the wife was so worried about his safety, that she did not let him go anywhere alone, even in their home. The suicide risk screening allowed the asQ'em team to collect this information and uncover that the patient was noncompliant with previously prescribed psychotropic medications, which put him at greater risk for harming himself.

Although none of the patients were at imminent risk, there were examples of past suicidal behavior that was highly relevant background information. For instance, it would be important for the medical team to know the history of the woman who impulsively took a bottle of acetaminophen four years ago, after a marital conflict, so in the future the team could ensure appropriate supports for the patient if they needed to deliver poor prognostic news.

A significant quandary arises from implementing suicide screening on a busy medical/ surgical hospital unit: Do patients who report past suicidal thoughts or attempts require an immediate psychiatric evaluation? Our data showed that screening provided an opportunity to recognize pressing concerns that have potentially serious consequences, but the cases were non-acute. Perhaps the follow-up question, "Are you having thoughts of suicide right now?", may be the most critical assessment question; a positive response to this question would signal a more acute situation that requires an immediate or "stat" psychiatric consult.

Moreover, in our sample, past suicide attempts occurred between 3 months and 25 years prior to screening. This wide range raises a difficult clinical question: does a patient who attempted suicide 25 years ago warrant the same psychiatric response as a patient who attempted 4 years ago? From examining the data, and speaking with the patients, suicide attempts exceeding ten years in the past seemed far enough in the past that, while still worthy of a discussion with the care team, did not warrant a full psychiatric evaluation. For example, the 4 patients who reported that they tried to kill themselves when they were 
young adults, 18 or 25 years ago, had developed better coping strategies over the past decade and did not require an urgent evaluation. Future research will need to further examine the association of "time since past suicide attempt" and current suicide risk.

\section{Limitations}

This was a quality improvement project with data collected from a convenience sample of hospitalized medical/surgical patients over the course of 9 weeks in a research hospital. All patients were participants in a clinical trial and may have been more willing to participate in a QIP. Therefore, the data may not be generalizable to other general medical settings. In addition, because of the pilot nature of this QIP, more intensive psychiatry services were made available than would be practical in a hospital setting. Furthermore, costs associated with utilizing nurses to screen for suicide risk were not assessed. Additionally, the goal of the asQ'em screening was to alert the clinician to the need for further evaluation of suicide risk; it was not intended to be predictive of completed suicide. It should be noted that the asQ'em tool, like other current instruments being used to assess for suicide in the medical setting, has not been validated on an adult medical population. Some hospitals use validated depression screens to assess for suicide; however, studies show that screening for depression may not be adequate for recognizing suicide risk in medical patients. ${ }^{23-26}$

\section{CONCLUSION}

Non-psychiatric nurses can feasibly screen adult medical/surgical inpatients for suicide without major disruptions to unit workflow. While the majority of medical/surgical patients will not have acute suicidal ideation, detecting suicidal thoughts and past behaviors can uncover meaningful clinical data.

\section{Acknowledgments}

\section{Funding /Support}

The Quality Improvement Project described in this article was supported by the Intramural Research Program of the National Institutes of Health and the National Institute of Mental Health; and by the NIH Clinical Center Nursing Department.

The authors wish to thank Ian Stanley, Deborah Kolakowski, Diane Aker, Felicia Andrews, Ann Marie Matlock, Kathleen Saimy, Jeasmine Aizvera, Gina Ford, Kim Cox, Janet Heekin, David Luckenbaugh, Bruce Steakley, and Jeanne Radcliffe; the physicians associated with the three inpatient units; and a special thanks to the bedside nurses, the clinical social workers, and all the patients for their participation.

\section{REFERENCES}

1. The Joint Commission: Summary Data of Sentinel Events Reviewed by The Joint Commission. http://www.jointcommission.org/sentinel_event_data_general/.

2. The Joint Commission: Sentinel Event Type - Event Type by Year. http:// www.jointcommission.org/assets/1/18/Event_Type_Year_1995_2Q2012.pdf.

3. The Joint Commission: Sentinel event data: root causes by event type. http:// www.jointcommission.org/Sentinel_Event_Statistics/.

4. Qin P, Webb R, Kapur N, Sorensen HT. Hospitalization for physical illness and risk of subsequent suicide: a population study. Journal of Internal Medicine. 2012

5. Furlanetto LM, Stefanello B. Suicidal ideation in medical patients: psychosocial and clinical correlates. General Hospital Psychiatry. 2011; 33:572-578. [PubMed: 21908051]

6. Fang F, Fall K, Mittleman MA, Sparén P, Ye W, Adami HO, Valdimarsdótter U. Suicide and Cardiovascular Death after a Cancer Diagnosis. The New England Journal of Medicine. 2012; 366:1310-1318. [PubMed: 22475594] 
7. Robson A, Scrutton F, Wilkinson L, MacLeod F. The risk of suicide in cancer patients: a review of the literature. Psychooncology. 2010; 19:1250-1258. [PubMed: 20213857]

8. National Action Alliance for Suicide Prevention Executive Committee. [last accessed Jan. 2, 2013] Clinical Care Intervention Task Force: Suicide Care in Systems Framework. http:// actionallianceforsuicideprevention.org/sites/actionallianceforsuicideprevention.org/files/taskforces/ ClinicalCareInterventionReport.pdf

9. Elderon L, Smolderen KG, Na B, Whooley MA. Accuracy and Prognostic Value of American Heart Association-Recommended Depression Screening in Patients With Coronary Heart Disease: Data from the Heart and Soul Study. Circ Cardiovasc Qual Outcomes. 2011; 4:533-540. [PubMed: 21862720]

10. Moscicki EK. Epidemiology of completed and attempted suicide: toward a framework for prevention. Clinical Neuroscience Research. 2001; 1:310-323.

11. Frankenfield DL, Keyl PM, Gielen A, Wissow LS, Werthamer L, Baker SP. Adolescent patients: healthy or hurting? Missed opportunities to screen for suicide risk in the primary care setting. Arch Pediatr Adolesc Med. 2000; 154:162-168. [PubMed: 10665603]

12. Pan YJ, Lee MB, Chiang HC, Liao SC. The recognition of diagnosable psychiatric disorders in suicide cases' last medical contacts. Gen Hosp Psychiatry. 2009; 31:181-184. [PubMed: 19269541]

13. Luoma JB, Martin CE, Pearson JL. Contact with mental health and primary care providers before suicide: a review of the evidence. Am J Psychiatry. 2002; 159:909-916. [PubMed: 12042175]

14. The Joint Commission: A follow-up report on preventing suicide: focus on medical/surgical units and the emergency department. Sentinel Event Alert. 2010; 46:1-4. [PubMed: 21089709]

15. Mills PD, Neily J, Luan D, Osborne A, Howard K. Actions and Implementation Strategies to Reduce Suicidal Events in the Veterans Health Administration. Jt Comm J Qual Patient Saf. 2006; 32:130-141. [PubMed: 16617944]

16. Horowitz L, Ballard E, Teach SJ, Bosk A, Rosenstein DL, Joshi P, Dalton ME, Pao M. Feasibility of Screening Patients with Nonpsychiatric Risk in a Pediatric Emergency Department: A Good Time to Talk? Pediatr Emerg Care. 2010; 26:787-792. [PubMed: 20944511]

17. King CA, O’Mara RM, Hayward CN, Cunningham RM. Adolescent Suicide Risk Screening in the Emergency Department. Academic Emergency Medicine. 2009; 16:1234-1241. [PubMed: 19845554]

18. Bridge JA, Goldstein TR, Brent DA. Adolescent suicide and suicidal behavior. J Child Psychol Psychiatry. 2006; 47:372-394. [PubMed: 16492264]

19. Rudd MD, Berman AL, Joiner TE, et al. Warning Signs for Suicide: Theory, Research, and Clinical Applications. Suicide Life Threat Behav. 2006; 36:255-262. [PubMed: 16805653]

20. Deming, WE. The New Economics for Industry, Government, Education. Cambridge, MA: Massachusetts Institute of Technology, Center for Advanced Engineering Study; 1993.

21. Horowitz LM, Wang PS, Koocher GP, Burr BH, Smith MF, Klavon S, et al. Detecting suicide risk in a pediatric emergency department: development of a brief screening tool. Pediatrics. 2001; 107:1133-1137. [PubMed: 11331698]

22. Horowitz LM, Bridge JA, Teach SJ, Ballard E, Kima J, Rosenstein DL, et al. Ask SuicideScreening Questions (ASQ): A Brief Instrument for the Pediatric Emergency Department. Arch Pediatr and Adolesc Med. 2012

23. Recklitis CJ, Diller LR, Li X, Najita J, Robison LL, Zeltzer L. Suicidal Ideation in Adult Survivors of Childhood Cancer: A Report from the Childhood Cancer Survivor Study. Journal of Clinical Oncology. 2010; 28:655-661. [PubMed: 19841325]

24. Razykov I, Ziegelstein RC, Whooley MA, Thombs BD. The PHQ-9 versus the PHQ-8 - Is item 9 useful for assessing suicide risk in coronary artery disease patients? Data from the Heart and Soul Study. Journal of Psychosomatic Research. 2012; 73:163-168. [PubMed: 22850254]

25. Walker J, Hansen CH, Hodges L. Screening for suicidality in cancer patients using Item 9 of the nine-item patient health questionnaire; does the item score predict who requires further assessment? Gen Hosp Psychiatry. 2010; 32:218-220. [PubMed: 20302999] 
26. Razykov I, Hudson M, Baron M, Thombs BD. Canadian Scleroderma Research Group. The Utility of the PHQ-9 to Assess Suicide Risk in Patients with Systemic Sclerosis. Arthritis Care Res. 2012 [Epub ahead of print]. 


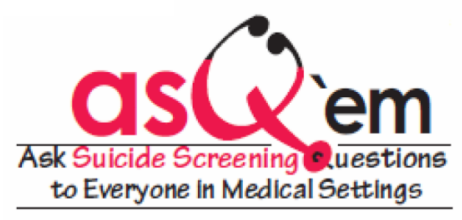

Suicide Screening Tool

Please say to the patient: "We are working to improve patient safety in the hospital. Sometimes, people with medical illnesses experience emotional distress and even have thoughts of ending their own life that they keep to themselves. We, as healthcare providers, believe we can help. Therefore, the next set of questions has to do with the sensitive topic of suicide. We are asking you these questions because of a new patient safety initiative and are currently asking these questions of all newly admitted patients on this unit."

1. "In the past month, have you had thoughts about suicide?"

$\square$ No If no, skip to question 2.

$\square$ Yes* If yes, say "can you please describe the thoughts you have had?" Write in:

2. "Have you ever made a suicide attempt?"

$\square$ No

$\square$ Yes*

- If yes, ask "when?"

- If yes, ask "how?"

* For any positive response

If yes to question 1 or $\mathbf{2}$, please ask "are you having thoughts of suicide right now?"

$\square \quad$ No

Yes If yes, say "can you describe the thoughts you are having right now?" Write in:

\section{IF YES TO ANY QUESTION:}

Please say to the patient: "Thank you for answering these questions. I am going to talk with your doctor and call someone who is trained to talk with patients about suicidal thoughts."

\section{IF NO TO ALL QUESTIONS:}

Please say to the patient: "That completes the suicide screening. Is there any feedback you would like to give us about being screened for suicide in the hospital?"

“Thank you for answering these suicide screening questions."

Figure.

The asQ'em Screening Tool 
Table 1

Patient Characteristics of Positive Screens

\begin{tabular}{|c|c|c|c|c|}
\hline ID & $\begin{array}{l}\text { Diagnostic } \\
\text { Category }\end{array}$ & $\begin{array}{l}\text { Age } \\
\text { Range }\end{array}$ & Sex & Description \\
\hline \multicolumn{5}{|c|}{ Screened Positive for Thoughts of Suicide in the Past Month } \\
\hline $1^{*}$ & Cancer & $18-39$ & Male & $\begin{array}{l}\text { In the context of physical symptoms } \\
\text { associated with cancer, patient reported } \\
\text { thoughts of suicide with a plan to step in } \\
\text { front of a train. }\end{array}$ \\
\hline $2^{*}$ & Cancer & $40-59$ & Female & $\begin{array}{l}\text { Patient reported thoughts of suicide in the } \\
\text { context of psychosocial stressors } \\
\text { (financial, legal) and pain. }\end{array}$ \\
\hline $3^{*}$ & Cancer & $40-59$ & Male & $\begin{array}{l}\text { Patient reported thoughts of suicide in the } \\
\text { context of frustration with repeated } \\
\text { inpatient hospitalizations. }\end{array}$ \\
\hline $4^{*}$ & Cancer & $40-59$ & Female & $\begin{array}{l}\text { Patient thought about suicide in the } \\
\text { previous week prior to a major organ } \\
\text { biopsy. }\end{array}$ \\
\hline 5 & Cancer & $40-59$ & Male & $\begin{array}{l}\text { Patient considered suicide after learning } \\
\text { he was disqualified from a research } \\
\text { protocol. }\end{array}$ \\
\hline \multicolumn{5}{|c|}{ Screened Positive for Past Suicide Attempt } \\
\hline $6^{*}$ & Cancer & $60-79$ & Male & $\begin{array}{l}2 \text { years ago patient jumped from a high } \\
\text { rise building while under the influence of } \\
\text { opiates and was severely injured. }\end{array}$ \\
\hline 7 & Cancer & $60-79$ & Female & $\begin{array}{l}25 \text { years ago patient overdosed on aspirin } \\
\text { after feeling like she neglected child care } \\
\text { responsibilities. }\end{array}$ \\
\hline $8^{*}$ & Cancer & $18-39$ & Male & $\begin{array}{l}3 \text { years ago patient took an overdose of } \\
\text { cold medicine after feeling anxiety and } \\
\text { pressure at school. }\end{array}$ \\
\hline 9 & Cancer & $18-39$ & Male & $\begin{array}{l}18 \text { years ago patient cut his wrist after a } \\
\text { break-up with a girlfriend, and the } \\
\text { pressure of two jobs and classes. }\end{array}$ \\
\hline $10^{*}$ & General Med/Surg & $40-59$ & Female & $\begin{array}{l}4 \text { years ago patient overdosed on } \\
\text { acetaminophen following marital conflict. }\end{array}$ \\
\hline $11^{*}$ & General Med/Surg & $40-59$ & Male & $\begin{array}{l}3 \text { months ago patient overdosed on pain } \\
\text { pills because he was in excruciating physical pain.. }\end{array}$ \\
\hline 12 & General Med/Surg & $18-39$ & Female & $\begin{array}{l}18 \text { years ago patient overdosed on } \\
\text { ibuprofen. }\end{array}$ \\
\hline $13^{* *}$ & Infectious Disease & $18-39$ & Female & $\begin{array}{l}18 \text { years ago patient overdosed on sleep } \\
\text { medication. }\end{array}$ \\
\hline
\end{tabular}

Note. No patient endorsed both suicidal thoughts and attempts.

Patient required full psychiatric evaluation.

**

Healthy volunteer for a clinical trial. 
Table 2

Patient Feedback Survey Responses from Phase I

\begin{tabular}{|c|c|}
\hline Patient experience & $\begin{array}{c}\mathbf{N}(\%) \\
{[\text { Total } \mathbf{N}=53]}\end{array}$ \\
\hline \multicolumn{2}{|c|}{ *How comfortable were you answering questions about suicide in general? } \\
\hline Very Comfortable or Comfortable & $46(87 \%)$ \\
\hline Neutral & $4(8 \%)$ \\
\hline Very Uncomfortable or Uncomfortable & $3(5 \%)$ \\
\hline \multicolumn{2}{|c|}{ Overall, how would you rate your experience of being screened for suicide? } \\
\hline Positive & $42(79 \%)$ \\
\hline Neutral & $10(19 \%)$ \\
\hline Negative & $1(2 \%)$ \\
\hline \multicolumn{2}{|c|}{ "I am glad the hospital is asking patients about suicide" } \\
\hline Strongly Agree or Agree & $45(85 \%)$ \\
\hline Neutral & $8(15 \%)$ \\
\hline \multicolumn{2}{|c|}{ "I felt like it was a burden to be asked about suicide" } \\
\hline Strongly Disagree or Disagree & $50(94 \%)$ \\
\hline Neutral & $2(4 \%)$ \\
\hline Strongly Agree or Agree & $1(2 \%)$ \\
\hline \multicolumn{2}{|c|}{ "Being asked about suicide made me angry" } \\
\hline Strongly Disagree or Disagree & $52(98 \%)$ \\
\hline Strongly Agree or Agree & $1(2 \%)$ \\
\hline \multicolumn{2}{|c|}{ *"The suicide questions made me feel anxious/worried/nervous" } \\
\hline Strongly Disagree or Disagree & $45(85 \%)$ \\
\hline Neutral & $7(13 \%)$ \\
\hline Strongly Agree or Agree & $1(2 \%)$ \\
\hline \multicolumn{2}{|c|}{ Have you been asked about suicide in a medical setting before? } \\
\hline No/Don't know & $34(64 \%)$ \\
\hline Yes & $19(36 \%)$ \\
\hline \multicolumn{2}{|c|}{ Do you think all medical/surgical patients in a hospital should be asked about suicide? } \\
\hline Yes & $43(81 \%)$ \\
\hline No & $5(9 \%)$ \\
\hline Don’t Know & $5(9 \%)$ \\
\hline
\end{tabular}

